\title{
Challenges of achieving accreditation to ISO 6789-2:2017 - for the calibration of hand torque tools
}

\author{
Matthew Gypps ${ }^{1}$ \\ ${ }^{1}$ Trescal Limited, Saxony Way, Yateley, Hampshire, GU46 6GT, United Kingdom
}

\begin{abstract}
In recent years hand torque tools have been manufactured and calibrated in accordance with the standard BS EN ISO 6789:2003. A number of flaws were identified with this standard and therefore a revised version BS EN ISO 6789:2017 was released.
\end{abstract}

The new standard required a significant increase in the number of measurements to be made which in turn created a risk to engineers, from repetitive strain injuries and a potential increase in cost to customers.

As the market leader for calibration with a high demand for accredited calibration, Trescal set the following objectives: minimise risk to Engineers, maintain a cost effective service and be the first non-manufacturer to obtain accreditation to the revised standard.

Significant investment in automated equipment not only reduced the risk of injury to engineers but also reduced calibration times and therefore cost. However the use of automated solutions for calibration of mechanical devices, created a number of challenges when trying to achieve accreditation.

Differences in the interpretation of the ISO standard by national assessment bodies resulted in variations of requirements and timescales for achieving accreditation throughout Europe.

Our presentation will highlight some of these issues.

\section{Introduction}

Hand torque tool technology and the associated calibration requirements have progressed significantly since the standard was last published in 2003. A number of flaws have also been highlighted within the method described in the previous version which is why a revision was required which resulted in it being divided into two parts:

- ISO 6789-1:2017: "Part 1: Requirements and methods for design conformance testing and quality conformance testing: minimum requirements for declaration of conformance" [1]: the method described within is very similar to the previous standard, but the requirement for "certificate of calibration" has been removed and replaced with a requirement for a "declaration of conformance" to ISO 6789-1:2017. 
- ISO 6789-2:2017: "Part 2: Requirements for calibration and determination of measurement uncertainty" [2]: a significant increase in the number of tool operations. Measurements of the effects of the use of interface adaptors and rotation of the output drive of the tool are all specified and required along with the need to characterise each tool type and record the findings.

This has increased the complexity of uncertainty calculation and the result recording application. Without innovation, the new standard significantly increases the time and effort required by an engineer.

We encountered various challenges in order to meet the requirements of the new standard whilst maintaining a cost effective service.

As the market leader for calibration with a high demand for accredited calibration, Trescal UK set a target of being the first non-manufacturer to obtain accreditation to the revised standard within the UK. This meant that a large amount of work was required to determine a suitable solution, build the evidence, submit the Extension to scope and obtain accreditation in a short time scale. Trescal UK achieved our target one year ahead of the deadline (March 2018).

Having to perform more than twice the number of operations during each calibration was going to increase work time and the manual handling/repetitive strain injury on the staff.

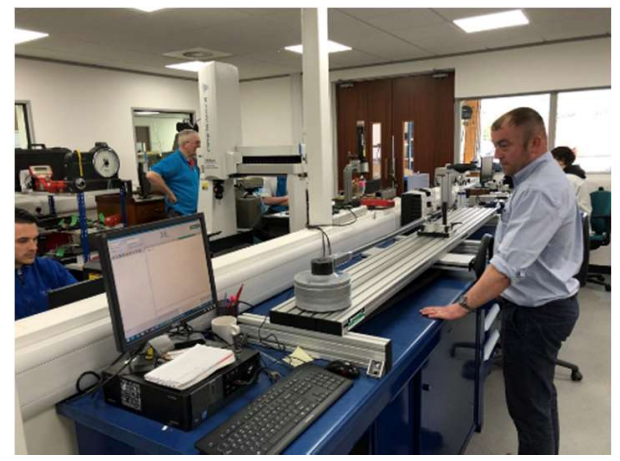

Fig. 1. Torque bench: Manual Loader

Through the use of automation Trescal have reduced these risks, improved accuracy and productivity enabling us to cope with higher demand for this service.

The projects involved 4 key people with skills in calibration, automation, programming and accreditation and staff at 3 UK locations.

Through discussion with our colleagues in other European countries it was evident that not only were there differences in the interpretation and application of the standard in other European counties but there appeared to be inconsistent deadlines and both errors and omissions during the translation of the standard which are still apparent today.

The next chapters will highlight some of these issues.

\section{Changes to the standard}

The most obvious change to the standard was the division of the standard into 2 parts as described in introduction. Some components of Part 1 also apply and are referred to in Part 2 and vice versa, so both parts must be considered together. 


\subsection{Major changes in Part 1}

\subsubsection{Torque range}

Section 5.1.3 specifies the torque range of the tool which is now "the lowest marked value to $100 \%$ of the maximum torque value". Previously tools were only calibrated to $20 \%$ of the maximum torque value irrespective of the markings on the tool. There was a risk that operators were using the tool in an unknown state of compliance below $20 \%$.

\subsubsection{Application of torque}

Section 6.2.1 states that the connection between torque measurement system and torque tool shall permit self-alignment so that parasitic forces and moments are minimized.

This is a change from the previous version whereby torque tools had to be mounted at $90^{\circ}\left( \pm 3^{\circ}\right)$ to the torque measuring device. This introduced side loads (parasitic forces) which influenced the torque measurement.

Section 6.2.4 introduces a varying time limitation for the applied torque to reach the target value (from $80 \%$ of target), dependant on the target torque value.

It is important to note that for torque screwdriver calibration a maximum time is also specified $(1.0 \mathrm{~s})$ this results in a $0.5 \mathrm{~s}$ window in which to reach the target value (from $80 \%$ of target) this presents a significant difficulty when using a manual loading device.

Section 8: The method described within part 1 is very similar to the previous version. However the requirement for "certificate of calibration" has been removed and replaced with a requirement in section 8 for a "declaration of conformance" to ISO 6789-1:2017.

\subsection{Major changes in Part 2}

In addition to the uncertainty of measurement of the calibration system, the new standard also takes into account the uncertainty of the torque tool itself.

Section 6.1 specifies the additional measurements to be performed which in addition to repeatability include:

6.2.2 Variation due to the reproducibility of the torque tool,

6.2.3 Variation due to the interface between the torque tool and the calibration system, comprising:

6.2.3.2 Variation due to geometric effects of the output drive of the torque tool,

6.2.3.3 Variation due to geometric effects of the interface between the output drive of the torque tool and the calibration system,

6.2.4 Variation due to the variation of the force loading point,

The standard does state that values can be determined statistically with a sufficient number of specimens (at least 10) or through manufacturer or 3rd party data so long as care is taken to ensure validation. 


\section{Accreditation}

Although ISO standards are often revised and superseded, it is often possible to continue to be able to perform calibration to a superseded standard. However, the UK assessment body, UKAS, stipulated that:

"All UKAS laboratories currently accredited to perform the calibration of hand torque tools to BS EN ISO 6789:2003 will be expected to transition to BS EN ISO 6789 - 2:2017" and that all laboratories will be expected to have transitioned to BS EN ISO 6789-2:2017 by 29 March 2019.

In addition UKAS stated that since there had been a "considerable technical revision" of the standard, that transition to BS EN ISO 6789-2:2017 would require an extension to scope (ETS) assessment. Assessment visits were to be arranged subject to assessor availability of which there were only 2 in the UK.

Since the introduction of ISO 6789:2017 within Trescal was likely to have significant impact on the UK service if not successful, the UK technical team worked closely with other Trescal Technical Teams across Europe. We quickly established that other accreditation bodes has not stipulated the same requirements and time scales as UKAS.

Several countries were permitted to continue to perform accredited calibration against ISO6789:2003, others were still awaiting advice from national accreditation bodies as late as November 2018.

\section{Challenges}

\subsection{Timescale}

The calibration services Trescal provide are often in line with ISO standards, so when these change, it is essential that we modify our internal procedures and process and be reassessed within given timescales in order to be able to continue to provide services against that standard.

ISO 6789:2017 was finally published on 31 March 2017. A deadline for transition (including closure of any improvement actions) by 29th March 2019 gave us 24 months.

Failure to meet this deadline would result in suspension of our accreditation and we would not be able to perform accredited Torque measurement we knew that we had to react quickly in order to avoid suspension of service. 
During this time we had to:

\begin{tabular}{|l|l|}
\hline Key Action & Estimated Time \\
\hline $\begin{array}{l}\text { Perform a gap analysis and develop a } \\
\text { transition plan Prior to submitting an } \\
\text { ETS }\end{array}$ & 2 months \\
\hline $\begin{array}{l}\text { Determine suitable equipment for 5 } \\
\text { accredited laboratories }\end{array}$ & 2 months \\
\hline $\begin{array}{l}\text { Purchase suitable equipment making } \\
\text { allowance for lead time, Factory } \\
\text { acceptance testing and commissioning }\end{array}$ & $3-4$ months \\
\hline Create procedures \& Uncertainty budgets & 1 month \\
\hline Create software & $1-2$ months \\
\hline Train staff and record cross checks & 2 months \\
\hline Submit Extension to scope & 3 months \\
\hline a/w assessment (5 locations) & $2-3$ months \\
\hline Resolve and submit improvement actions & 1 month \\
\hline Contingency (10\%) & 2 Months \\
\hline \multicolumn{1}{|c|}{ Total Estimated Time } & $\mathbf{2 2}$ Months \\
\hline
\end{tabular}

Table 1. Planning: road to accreditation according to the new version

As you can see from our original estimates, we had a potential 22 month project to achieve in 24 months. A considerable amount of this time was dependent on 3rd parties and therefore it was essential that project was started without delay.

\subsection{Number of operations}

The ISO 6789:2003 standard required only 15 measurement in each direction to calibrate a hand torque tool.

ISO 6789:2017 requires additional measurements in order to characterise each tool type and record the findings. This results in an additional 120 tests per tool in each direction.

Without innovation, the new standard significantly increases the time and effort required by an engineer. A technician calibrating 10 tools per day may need to perform an additional 1200 operations which on a $1000 \mathrm{~N} \cdot \mathrm{m}$ torque wrench would require a significant amount of effort, potentially leading to injury.

\begin{tabular}{|l|c|c|}
\hline \multicolumn{1}{|c|}{ Measurement } & \multicolumn{2}{|c|}{ Number of tests } \\
\hline & ISO 6789:2003 & ISO 6789:2017 \\
\hline Repeatability & 15 & 15 \\
Reproducibility & $\mathrm{n} / \mathrm{a}$ & 20 \\
Geometric effects of output drive & $\mathrm{n} / \mathrm{a}$ & 40 \\
Variation of interface & $\mathrm{n} / \mathrm{a}$ & 40 \\
Variation of Torque Loading Point & $\mathrm{n} / \mathrm{a}$ & 20 \\
\hline Total & $\mathbf{1 5}$ & $\mathbf{1 3 5}$ \\
\hline
\end{tabular}

Table 2. Comparison with the old and new version of the standard in term of number of tests 


\subsection{Time of calibration}

If we assume an average calibration time of 15 minutes for a calibration in accordance with ISO 6789:2003, then a calibration to ISO 6789:2017 could take as long as 2 hours to complete manually.

\subsection{Test Equipment}

It was decided that an automated solution was required to minimise the risk of injury and length of calibration time. We therefore had to either modify the existing systems or invest in new equipment.

The stipulation that the calibration system should self-align meant that the current calibration systems in use were not suitable for calibration against the new standard.

Several automated solutions were on the market or due for launch in the near future but only one product fulfilled our full requirements.

Trescal therefore invested in 5 Stahlwille Perfect Control systems with Torkmaster software.

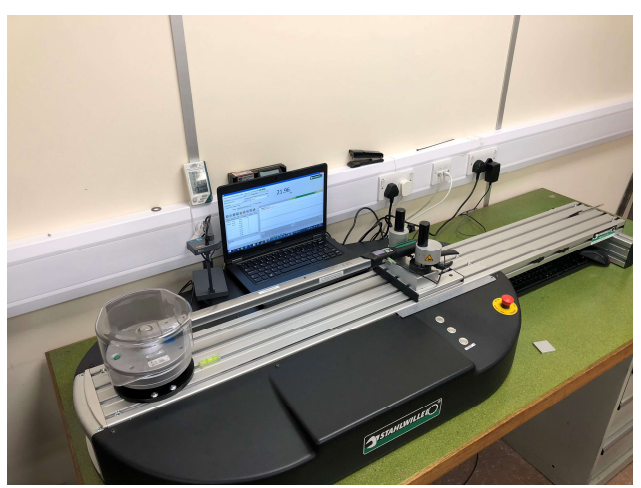

Fig. 2. Stahlwille Automated loader bench

\subsection{Software}

Due to a difference in the interpretation of the standard between German and UK accreditation bodies, the Stahlwille Torkmaster software did not initially fulfil all UK requirements for continued use against ISO 6789:2003. We worked closely with the manufacturer to specify and achieve the required modifications to the software and also developed our own solution as an interim measure.

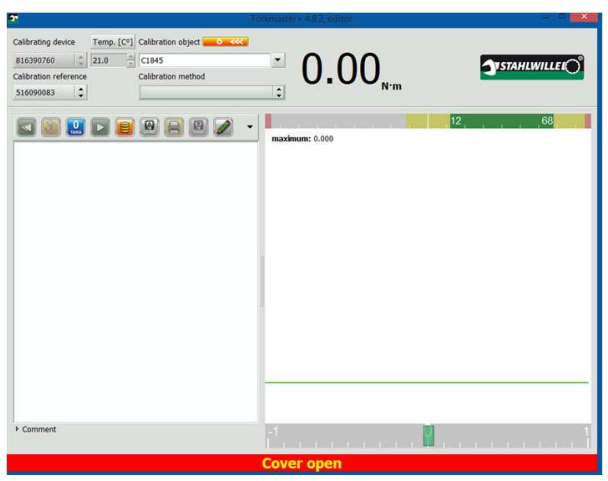

Fig. 3. Software visualization 


\subsection{Other country variances}

\subsubsection{Part 1 certification}

In the UK we are not permitted to offer a certificate of calibration against part 1 of the standard, only a declaration of conformance can be issued. However in France they are accredited to part 1 and part 2 and can issue a certificate for either.

\subsubsection{Statistical analysis}

The position is unclear. The standard states that uncertainty contributions "can be determined statistically with a sufficient number of specimens (at least 10).”

In the UK the Trescal interpretation was the data from 10 different units of the same type. However in France, collection of 10 sample data sets from the exact same tool is acceptable. Other assessors suggest that each tool must be considered uniquely any tool may have been subject to misuse.

At present, we have no firm conclusion but intend to prove our chosen method statistically.

\subsection{Calibration failures}

ISO 6789:2003 stipulated that measures were made no lower than $20 \%$ of the tool range irrespective of the markings on the tool. Since ISO 6789:2017 states that calibration will be from "the lowest marked value" we are now testing some older tools much lower than previously and are finding tools that historically passed (at 20\%) are now failing or have a very high uncertainty due to poor repeatability. This is causing some concern and confusion with customers but does emphasise the risk in the design or manufacture of some tools to the old standard.

\subsection{Certification}

Standards: ISO 6789:2017 requires a measurement uncertainty interval (W') for each transducer used in the calibration system. Historically this was not provided on calibration certificates and therefore BS 7882:2017, "Method for calibration and classification of torque measuring devices" [3], was issued and Trescal UK also had to achieve accreditation to this standard at the same time as achieving accreditation to ISO 6789:2017.

Reporting results: In order to state compliance of a torque tool, the calculated measurement uncertainty interval (W') has to be compared to the measurement uncertainty interval provided by the customer. As this has previously not been required, most customers are not familiar with this requirement and have often been unable to provide this data.

\section{CONCLUSION}

Whilst the introduction of ISO 6789:2017 should result in an improved quality, and a more reflective calibration of hand torque tools, it has introduced a number of challenges.

When we first understood the timescale to achieve accreditation in the UK we felt that this was adequate. However due to the significant changes in the procedure and the steps required we quickly realised, even with basic estimates, this would be hard to achieve. Thankfully with a talented and hardworking team, a close relationship with the manufacturer and submitting our extension to scope before others, we were able to complete this project ahead of schedule. 
Unfortunately the timescale and cost of investment required has resulted in a significant reduction in the number of accredited laboratories within the UK. Whilst in the immediate future, calibration of older hand torque tools to the ISO 6789:2003 standard may be acceptable, once these tools are phased out and replaced with items built to the new standard, customers may not be provided with an appropriate calibration service from non-accredited providers thus increasing the risk of using non-compliant tools.

The use of motorised or automated calibration systems is essential for minimising the risk to operators and reducing calibration times in order to provide a cost effective service. The investment in this equipment has dramatically improved our repeatability of measurement when calibrating hand torque tools and allowed us to maintain a cost effective service for our customers.

Differences in the interpretation and application of ISO standards between countries should be addressed.

The ethos behind international standards is that items are manufactured and calibrated to the exact same requirements worldwide. If assessment bodies permit variance between countries it can increase risk to the end users of the tool or users of products manufactured using that equipment. Timescales of deployment and the view on continued use of withdrawn standards must also be aligned and possibly form part of the content of each standard. 


\section{References}

1. EN ISO 6789-1:2017, Assembly tools for screws and nuts - Hand torque tools - Part 1: Requirements and methods for design conformance testing and quality conformance testing: minimum requirements for declaration of conformance

2. EN ISO 6789-2:2017, Requirements for calibration and determination of measurement uncertainty

3. BS 7882:2017, Method for calibration and classification of torque measuring devices 